\title{
From Cloud Computing to Fog Computing (C2F): The key technology provides services in health care big data
}

\author{
Babur Hayat Malik, Sadaf Nawaz Cheema*, Iqra Iqbal, Yasar Mahmood, Majid Ali, and \\ Ahmad Mudasser
}

Department of CS and IT, University of Lahore, Gujrat Campus, Pakistan

\begin{abstract}
Because of the broad utilization of web-based social networking, data is produces by the fast increment. Big Data is giving the office to accumulate, store, oversee and examine information in colossal volume that is produced through the healthcare system. Cloud Computing is an advancement too that insures the fulfillment of IT requirements in a suitable way by providing the cloud-based environment for medical field. Storage is an immense issue for $\mathrm{BD}$, volume of data is huge, this issue may resolve with the help of cloud computing by providing the storage space for data and processing mechanism as well. This paper presents these thoughts with respects to medicinal services. It tells regarding the points of interest, yet in addition challenges conveyed by Big Data to this field. It additionally talks about the idea of fog computing, some advantages of edge computing on cloud computing and deliberate the architecture of fog computing for healthcare and services provides by that architecture.
\end{abstract}

\section{Introduction}

Healthcare plans to retain and enhance human health by means of analysis, treatment and disease counteractive action. As inspired by patient care, inspecting, organization and necessities to comply with principles and controls, it generates enormous measures of information - Big Data consistently BD can possibly Support numerous medicinal and healthcare tasks, including clinical choice help, disease surveillance and population health management. Cloud computing can diminish the expenses of computerization and framework support, then enhancing operational effectiveness and client access. A tremendous amount of data has generated, by the Innovations in the OMICS-fields (proteomics, genomics) for processing and storing purpose. Different types of data are collected for different purposes and then converted in digital form. To achieve these requirements cloud computing is a good solution. Clouds guarantee points of attention in unique assets like computing power or capacity edges, pervasive approach to resources whenever from anywhere, and high flexibility and versatility of resources by the different benefits adaptation of cloud computing is increasing in many business domains.

Big Data in healthcare services is overpowering a result of its volume along with in view of the decent variety of information writes and the speed on which data must be

*Corresponding author: sadaf_cheema139@yahoo.com 
access. 3G/4G/5G are the technologies used in the cloud and the modest mobile devices. Another current innovation is cloud computing (see Fig. 1) by using that you can access information at any time and different organizations could be used this as well as individual users for the betterment in performance. NIST define the concept of cloud computing as: a paradigm for emboldening extensive, helpful, on-request arrange approach to a common pool of configurable managing assets can be provisioned and discharged quickly with minor administration exertion or specialist association collaboration [1].

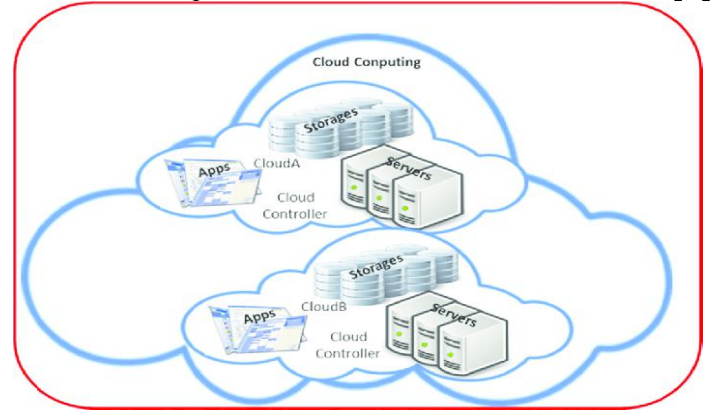

Fig. 1. Cloud computing concept.[2]

Clouds promise advantages in dynamic resources like computing power or storage capacities, ubiquitous access to resources at any time from any place, and high flexibility and scalability of resources [3].Because of the heterogeneous sources and huge volume, healthcare data is more complex. As big data is a new pattern, same way it's new in the field of biomedical informatics. To achieve IT need in true sense in healthcare sector cloud computing is a great roadmap. It is not possible for cloud to give the sensing facility and many devices use sensor technology, those are utilizing in many hospitals. Another issue is that, due to data confidentiality, some rules do not give permission to store the data outside the boundary of that hospital. Fog computing plays the role of bridge to overcome the gap for the sensors application in hospitals. A term often used synonymously is edge computing, describing tasks that are placed at the edge of the network in contrast to the cloud [4].

The literature covers the topic of cloud computing in healthcare from a variety of viewpoints. Further, we need to recognize areas those are facing problems inside the healthcare field where cloud computing applications have for the most part been conferred. The paper is containing the following different parts. In Sec-I, presently we will show the model of Big Data, Cloud computing and list the key characteristics. In Sect-II, we discuss some of the drifts and obstacles of cloud computing in healthcare. In Sect. III, we show the yearly distribution of publications according to MEDLINE Results. In Sect. IV we provide a big picture of the fog computing, its characteristics and how is it overcome the issues in the healthcare sector. In Sect. V discussion regarding the fog architecture for healthcare and its services. We determine with an indication of the existing condition of study, and also determine outline for further research for employing fog computing in healthcare.

\section{Background}

\subsection{Cloud computing}

Cloud computing could be a quickly developing advancement that has developed itself inside its following time industry. Effective design of cloud services can execute extensive computing tasks and period orchestrates of IT works from storing and calculation to database and function facilities. The new cloud computing technology promises to satisfy the IT needs in a more favourable way [5]. Different companies use cloud computing to process and analyse huge volume of datasets. Furthermore, cloud facility suppliers have 
started to combine frameworks for similar processing of data in their packages to support cloud resources for the access of user. Cloud computing model allows appropriate network access too many aligned computing sources that can be speedily provisioned and freed with least managing effort. NIST cloud framework also defines five key attributes, three service models, and four deployment models [6]. Cloud computing model based on of PaaS, SaaS, and IaaS.

\subsection{Big data definitions and characteristic' $s$ in Healthcare}

Since being authored in 1997 by NASA specialists, the term BD has been reclassified over and over amid the years [7]. According to a 2013 Commonwealth of Australia report, about $90 \%$ of data today was created in the last 2 years [8]. Every day, all kinds of data sources generate 2.5 quintillion bytes of data [9]. Big data is recognized as a multidisciplinary information processing system. Areas of business, government, media, and in particular healthcare, are increasingly incorporating big data into information processing systems [8].

- "Data of a very large size, typically to the extent that its manipulation and management present significant logistical challenges; also, the branch of computing involving such data" (Oxford English Dictionary)

Most primary data is created and stored by health services providers, including general practitioner doctors, specialists and surgeons, public and private hospitals and clinics [10].The attributes of BD can be combined by 7 Vs [7], which are outlined in Fig.2.

i. Volume: Huge amount of data is already gathered from millions of patients and stored electronically to increase the efficiency of healthcare services and provide many research opportunities. Storage is a big problem.

ii. Variety: Because of heterogeneity in data sources, it is difficult to decide a specific pattern of data. It includes semi structured or unstructured data in healthcare sector. For example, text messages, online games, blogs, and social media generate different types of unstructured data through mobile devices and sensors [8].

iii. Velocity: The term Velocity alludes to the speed with which information is created. The age of state-of-the-art comes about because of ongoing or close constant preparing is vital, for instance, in clinical choice help, for having the capacity to settle on the correct choices quickly [11]

iv. Veracity: Data veracity concerns with safety of the patient, but at the same time there is a threat of less quality, wrong and unstructured data [5].

v. Variability: Variability refers to interpretable factor the data. For instance, advanced calculations are fundamental with a specific end goal to deduct the right significance of web-based social networking remarks.

vi. Visualization: Visualization alludes to the meaningfulness about the data introduction, which needs various geographical and worldly limitations and connections among them.

vii. Value: Value denotes to the prospect of making new information and financial incentive by misusing the information.

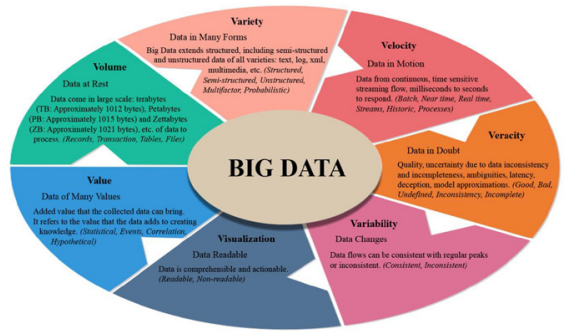

Fig 2. Big data characteristics [7]. 


\section{Research on big data and cloud computing in Healthcare}

\subsection{Year of publications}

This section the shows the yearly statistics about the publications according to the MEDLINE results. Figure 3 depicts that publications on big data and cloud computing in healthcare sector. It also tells the yearly distribution of articles about the topic. Management of big data and cloud computing architecture devices are expensive ones and they may take long time for adoption. Moreover, these are latest technologies that's why they require some time for the proper working and become the part of the existing system.

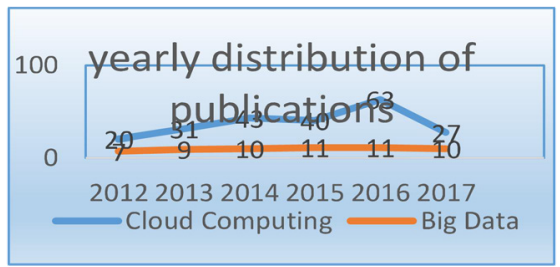

Fig.3. Yearly distribution of published articles.

\section{Limitations and obstacles of cloud computing}

Security threats: Organizations are having sensitive data that is more confidential. Customers have to trust on the cloud service provider for the security of their data only.

Technical Hindrance: In cloud environment, data is on cloud instead of local system. That's why, there is essential requirement of high speed internet. Because if high speed connection is not available then there must be technical issue especially in bulk uploading.

Data lock-in: The absence of standard APIs confines the relocation of utilizations and administrations between clouds. With the ascent of cloud, the issues of Data movability, movement and seller secure circumstance will increment.

Data location: For the security of data and information about clients, important thing is that where is data located? Secure geographical location is necessary.

Recovery and back-up: Backup of data is very important in case of any disaster.

\section{Need of fog computing}

However, the IT industry giving benefit and solutions of the problems to almost all fields, healthcare sector is also one of them. Still, cloud models is not suitable for critical applications because too has different problems like extreme bandwidth restraints and random response time [12]. Fog Computing is a big alternative solution for the achievement of these requirements. Fog and edge computing in general is an emerging platform that provides computational, storage, and control resources in an intermediate layer between end-user devices and cloud computing datacenters [13].

The word fog computing was at first began by industry as an allegory for the fundamental design thought behind it: fog is anywhere close to the cloud and the ground, wherever the clients' means are found. A word frequently utilized synonymously is edge processing, portraying assignments that are put at the side of the network as opposed to the cloud. According to ETSI's terminology [14] the view of internet service providers, edge is the boundary of the operative's network, similar for example a station based on LTE. According to our interpretation of fog includes these two perspectives. Topology is the core feature of fog computing. Access points, network gateways and routers can be cohesive together with the standard network functions as the resources of the fog computing. There 
is a unanimity about fog computing, it is not the alternative of the cloud computing, yet rather see it as an impeccable partner or an expansion of it.

A. Reduced Latency: With the contrast of cloud based architecture device, fog computing gives fast response and overcome the latency due to the shorter physical path.

B. Eliminate Delays: Eliminate data transfer delays by reducing the distance from datacenter to client. For example, with the fog, some of the processing can be taken place at routers rather than transmitting all data to the cloud [15].

C. Privacy: With the contrast of cloud based architecture device, fog computing overcome the data promulgation by analyzing the confidential and sensitive data on local gateway instead of data center beyond the control of the user.

D. Energy Efficiency: Fog computing uses sensor devices which saves the energy by different ways. Gateways provide communication proxies, it improves the duration of sleep cycle and then other services can be loaded from the battery-driven nodes [16].

E. Bandwidth: With the contrast of cloud based architecture device, fog computing shrink's data size that is moved to the data centers.

F. SCALABILITY: Fog computing enhance the adaptability of a system. Local computation diminishes the heap from more central sources, and be extended as required.

G. DEPENDABILITY: System dependency can be increased by two ways by using fog computing to decrease the dependency of the availability of network connection, computation is performing nearer to the sensor nodes

\section{Discussion}

Besides, all its potentials, it is in its initial stage in the healthcare and not implemented completely.

\subsection{Fog computing features and solutions to different problems}

Fog computing makes the architecture more robust because it is sited between IoT sensors and cloud data centres and throw-outs all problematic and content. Fog resolves following IoT related constraints

A. Extensive bandwidth requirements: Supporting billions of mechanisms IoT ecosystems makes data-oriented problems. Different bleak datasets are collected by end nodes and submitted for processing to the cloud. This kind of approach is worthless. Barren datasets are increasing continuously, so pre-processing is necessary. Data mining would be advantageous to overcome such issues [12].

B. Necessity for decreased latency and autonomous operation: It is needed to decrease independent processes and latency rate because interconnected nodes are continuously increasing, cloud services will be interrupted in case of improper connectivity. The beginning of 5G [17] will resolve our currently compromising networking issues but existing model requires redundancy and robustness.

C. Enhanced consistency and security fundamentals: If additional data travels throughout the network there would be more errors, these errors can't be tolerated for the functioning.

\subsection{The implemented fog}

Fog computing architecture is in its simple form shown in Fig. 4. According to this framework cloud and end devices are having fog between them. The Fog are heterogeneous in nature, from the top of the line servers, edge switches, get to focuses, set-top boxes, to the end gadgets, for example, cell phones, apple watch, and sensors and so forth. 


\subsection{The duty of fog computing}

As already discussed, primary role of fog computing is that to provide the facility of service on network edge. In fog environment, all the devices are attached on the network edge which further connect with the cloud, as shown in figure.5. Fog is just like a third party between cloud and devices. Fog receives data from devices and perform some processing upon then transmit data to the cloud. The Cloud provide the storage for big data, data mining and some calculated results, some feedbacks to the fog. This is the duty of the fog to command the end devices for the receiving of the calculated results and control them. With regard to the location awareness, the Fog architecture can be used to build models enhanced by this information, or to produce specific outcomes (like the location of injured subjects or the movement of crowds inside an ambient) [18].

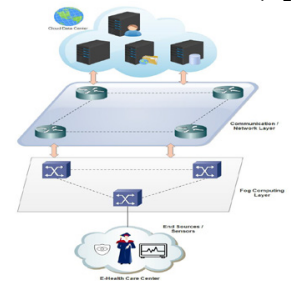

Fig. 4. Fog computing architecture.

A. Switching Network: The Fog needs to help numerous conventions, for example, ZigBee, WiFi, 2G/3G/4G, WiMax, 6LOWPAN et cetera. In any case, the Cloud just backings TCP/IP.

B. Pushing Service: The pushing service incorporates accepting the information and transferring the information. The end devices transfer information to the Fog after they produce the information.

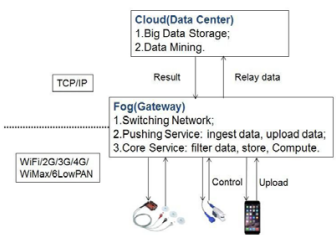

Fig. 5. Fog computing architecture with specific purpose [19].

C. Core Service: Fog must perform storage and processing. The major task performed by the fog is that to receive the data at local end then perform the computations on that and then transferred.

\section{Conclusion}

Healthcare system can be operative and proficient with the help of BD. They can be used for different operations from disease managing and restraint to medical research, and practice to insights which proof healthcare providers in making more in time and well conscious results about the population they are handling. It can be possible by using the storage and processing capacity of cloud computing and so the combination of BD and cloud computing is often promoted as a recipe for success. In fact, our review demonstrates that calculation is an essential component in all unavoidable health services applications, and that these errands frequently should be executed somewhere close to the sensors and the cloud. It likewise demonstrates that there is potential for calculation at all system levels. Thus, an appropriate model to rally the needs of healthcare. Fog computing errands can clean and filter data to facilitate preserve privacy issues or lessen the load on the network. The Fog as a binding together design is to execute some portion of server assignments at 
the edge of the server and, at long last, empower the new advancement of the dormancy delicate IoT applications. In future works, the nearby putting away and registering procedures, actualizing the fog computing in the BAN, vehicle systems and different fields will be contemplated. This study and analysis is an indication into this heading, outlining the wide traverse of, prerequisites in future health services and the variety of fog computing tasks.

\section{References}

1. P. Mell and T. Grance, "The NIST Definition of Cloud Computing Recommendations of the National Institute of Standards and Technology," Nist Spec.., vol. 145, 2011.

2. L. A. Tawalbeh, R. Mehmood, E. Benkhlifa, and H. Song, "Mobile Cloud Computing Model and Big Data Analysis for Healthcare Applications," IEEE Access, 2016.

3. L. Griebel et al., "A scoping review of cloud computing in healthcare," BMC Medical Informatics and Decision Making. 2015.

4. F. A. Kraemer, A. E. Braten, N. Tamkittikhun, and D. Palma, "Fog Computing in Healthcare-A Review and Discussion," 2017.

5. T. Ermakova, J. Huenges, K. Erek, and R. Zarnekow, "Cloud Computing in Healthcare - a Literature Review on Current State of Research," no. 1.

6. V. K. Nigam and S. Bhatia, "Impact of Cloud Computing on Health Care," pp. 2395-56, 2016.

7. D. C. ADRIANA ALEXANDRU1, CRISTINA ADRIANA ALEXANDRU2 and E. TUDORA1, "Healthcare, Big Data and Cloud Computing," WSEAS Trans. Comput. Res., vol. 4, pp. 126-131, 2016.

8. R. Hermon and P. A. H. Williams, "Big data in healthcare: What is it used for?"

9. H. Chen and F. Zhongchuan, "Multilabels-Based Scalable Access Control for Big Data"

10. I. A. T. Hashem, I. Yaqoob, N. B. Anuar, S. Mokhtar, A. Gani, and S. Ullah Khan, "The rise of 'big data' on cloud computing: Review and open research issues," 2015.

11. W. Raghupathi and V. Raghupathi, "Big data analytics in healthcare: promise and potential," Heal. Inf. Sci. Syst., 2014.

12. V. Palanisamy and R. Thirunavukarasu, "Implications of big data analytics in developing healthcare frameworks â€ "A review," 2017.

13. [14] O. Akrivopoulos, I. Chatzigiannakis, C. Tselios, and A. Antoniou, "On the Deployment of Healthcare Applications over Fog Computing Infrastructure," in 2017 IEEE 41st Annual Computer Software Conference (COMPSAC), 2017.

14. M. Ianculescu, A. Alexandru, and E. Tudora, "Opportunities brought by big data in providing silver digital patients with ICT-based services that support independent living and lifelong learning," in International Conference on Ubiquitous and Future Networks, ICUFN, 2017.

15. R. Deng, R. Lu, C. Lai, and T. H. Luan, "Towards power consumption-delay tradeoff by workload allocation in cloud-fog computing," in IEEE International Conference on Communications, 2015.

16. B. Farahani, F. Firouzi, V. Chang, M. Badaroglu, N. Constant, and K. Mankodiya, "Towards fog-driven IoT eHealth: Promises and challenges of IoT in medicine and healthcare," Futur. Gener. Comput., 2017.

17. A. M. Rahmani et al., "Exploiting smart e-Health gateways at the edge of healthcare Internet-ofThings: A fog computing approach," Futur. Gener. Comput. Syst., 2018.

18. L. Cerina, S. Notargiacomo, M. G. Paccanit, and M. D. Santambrogio, "A fog-computing architecture for preventive healthcare and assisted living in smart ambients," in RTSI, 2017.

19. Y. Shi, G. Ding, H. Wang, H. E. Roman, and S. Lu, "The fog computing service for healthcare," 2015 2nd Int. Symp. Futur. Inf. Commun. Technol. Ubiquitous Healthc., pp. 1-5, 2015. 